\title{
Depiction of semi-algebraic and semi-analytic set by means of particle swarm optimization
}

\author{
Ichio Kikuchi and Akihito Kikuchi*
}

December 27, 2018

\begin{abstract}
We present an algorithm of particle swarm optimization (PSO) which can depict the boundary of semi-algebraic or semi-analytic set. The algorithm imitates physical phenomena: the pair-annihilation of particle and antiparticle (or electron and hole). The pairs of particle and antiparticles are attracted by the boundary of the boundary of semi-algebraic or semi-analytic set, led by a special penalty function. The accumulation of these pairs around the optima will act as the "group best set" in the PSO algorithm (which also attracts the other pair) and it finally depicts the boundary.
\end{abstract}

\section{Introduction}

In recent decades, the algorithms, known as the particle swarm optimization (PSO), which imitate the behavior of flocks of birds or schools of fishes, has shown the efficiency as the optimization technique in various topics[1, 2, 3, 4, 5, 6, 7, 8, 9, 10]. Based on these developments, in this article, we construct a variant of PSO algorithm which enables us to depict the form of the boundary of semi-algebraic or semi-analytic set. The algorithm is an imitation of physical phenomena, such as the pairannihilation of particle and the antiparticle, or the formation of exciton between electron and hole. In the physical world, The members of the particle-antiparticle pair have the positive and negative charges, and they attract with each other, annihilate themselves of forms a certain bounded state. If we can give such pair-forming property to the sample particles in the optimization algorithm, and if we can assemble the bounded pairs to the boundary of the semi-algebraic or analytic set, it means that we can depict the boundary.

Concerning the depiction of the semi-algebraic set, the cylindrical algebraic decomposition (CAD) is the practicable algorithm in the symbolic computation[11, 12, 13, 14, 15]. In this algorithm, the variables are one by one eliminated from the set of the polynomials and sign conditions (which

*A.K. is the corresponding author: akihito_kikuchi@gakushikai.jp 
describe the semi-algebraic set), in order to generate the polynomials in lesser dimensions sequentially. Such eliminations of one variable are executed by means of the computation of the discriminant (to which the pairs of two polynomials are submitted). This process is called "the projection" and it is done until one obtains the set of single variable polynomials. Then the computation arouses itself through "the lifting process". At first, at the bottom stage of the projection, the real roots of the single-variable polynomials are computed. Thus the one-dimensional space is divided by the small segments by these roots. The algorithm then rises to the upper level of the projection, that is to say, in the two-dimensional real Euclidean space. This two-dimensional space is divided by the small cells; these cells are depicted by the segments in one-dimensional space and the zeros of the polynomials of two variables. Now the real-root findings are again necessary for the set of polynomials of two variables; in effect, to these polynomials, the value of representative sample points at the one-dimensional segments are substituted, and the root findings are the computations in the one-dimension. One should in inspect whether the algebraic set defined by the polynomial actually would cut and separate the real two-dimensional space. The algorithm again rises to the upper level of the projection, now entering into the three dimensions; it again divides the three-dimensional spaces into the small cells... In this way the lifting is done until the initial N-dimensional space is decomposed into the small cells; the boundaries of which are represented by the multitudes of polynomials (which are generated and accumulated by the projection in addition to the initial polynomials). Throughout the projection and the lifting, the crossings and the critical points in the geometrical manifolds depicted by the whole of the generated polynomials are listed up without omission. The algorithm carefully constructs the cell decomposition so that the sign-condition of the initially given polynomials (to be positive, zero or negative) is invariant inside each of the cells. One can, therefore, look for and connect the cells which shall satisfy the prenex formula with quantifiers (such as "Exists" or "For all" which decorate the polynomial equations and inequalities) so that one can obtain the formula, represented by polynomials with sign-conditions, and without quantifiers (the quantifier elimination: $\mathrm{QE})$.

Although CAD and QE are in theory very attractive, in practice they are always hindered by the explosive growth of the computational complexity according to the size of the problem. In N-dimension, the computational complexity scales as the double exponential of N. So in the present computational architecture, the brute-force reinforcement of the CPU power or the memory size is not the effective tactic. This hardship originates from the excessively rigorous way of symbolic computation: one may avoid it by a less rigorous way so that one can tackle to problems of larger sizes. The PSO might be a candidate of such an expedition. The computational complexity is proportional to the dimension of the problem. The swam of the sampling particle could fill the semi-algebraic/analytic set, once the equations and the inequalities are given. In this article, we will investigate the capability of PSO as such an expedient way. 


\section{Algorithm description}

Let us briefly review the standard PSO algorithm. The particles move in a way analogical to the behavior of a group of flying birds or other living creatures. They act as a group so that they should arrive at an optimized state in the end. In the algorithm of PSO, the positions of the birds are replaced by the set of particles in the Euclidean space. The target of the group is also represented mathematically by means of single- or multiobjective functions. The time-evolution of the particle system is executed in the discrete time steps, similar to the conventional molecular dynamics (MD) simulation the with stochastic flavor; each particle will be attracted to the personal best point $p_{\text {best }}$ (with respect to the objective function) and the group best point $g_{b e s t}$; the velocity of the particle $v^{j}$ is curbed by the frictional parameter and every member of the group will cease to move and assembles at the unique best position.

$$
\begin{gathered}
v^{j}=w \cdot v^{j}+C_{1} \cdot r_{1} \cdot\left(p_{\text {best }}^{j}-s^{j}\right)+C_{2} \cdot r_{1} \cdot\left(g_{\text {best }}^{j}-s^{j}\right) \\
s^{j}=s^{j}+\chi \cdot v^{j}
\end{gathered}
$$

The parameter $w$ is the inertia which leads the group to a halt in the end; $\mathrm{c} 1, \mathrm{c} 2$ and $\chi$ are the parameters which determine the extent of the jump of the velocities and the positions from one step to the next; the alternation of the velocity and the positions are also regulated by random numbers r1 and $\mathrm{r} 2$.

The PSO algorithm can be applied to the determination of the tradeoff curve or Pareto boundary. In this type of computation, the personal best or the group best cannot be determined as the unique point. Instead, the best points shall come and nest upon curves or surfaces. Thus the PSO algorithm tries to extend the frontier of the groups; the frontiers are updated again and again by the pioneers of the sample particles, which arrive at the current best positions (in the sense that the particle is not dominated by the others with respect to the multi-objective function). The frontier always attracts the swarm and extend itself by the accumulation of pioneers until it arrives at the limit of the growth.

The modification in our proposal from the usual PSO is summarized as this.

- Sampling pair We use the pairs of a particle (or electron) and an antiparticle (or hole), which will be attracted with each other, instead of single particles, in order to do the PSO sampling.

- Initialization The sampling pairs are distributed randomly in the euclidean space under the consideration. It is convenient that one of the pair (say, the particle) is located inside the semi-algebraic or semi-analytic set and the other is outside.

- The penalty function: type 1 In order to represent the feasibility of the equations and the inequalities, one may use this function:

$$
\Theta\left(X_{A} ; X_{h}\right)=0 \text { or } 1
$$

$\Theta\left(X_{A} ; X_{h}\right)$ should 0 or 1 , when a particular condition with respect to the position is satisfied or not. 
- The penalty function: type 2 The pair (represented by the duplicated coordinate $\left.\left(X_{P}, X_{A}\right)\right)$ should approach closely from one to another at the end of the optimization. The penalty can be represented by the function of the distance, with respect to the coordinates $X_{P}(i)$ and $X_{A}(i), i=1 . . N$, such as:

$D\left(X_{P}, X_{A}\right)=|| X_{P}-X_{A}||=\left|X_{P}(1)-X_{A}(1)\right|+\left|X_{P}(2)-X_{A}(2)\right|+\ldots+\left|X_{P}(N)-X_{A}(N)\right|$

Or one can use this form:

$D\left(X_{P}, X_{A}\right)=\left|D_{\text {min }}-\right| X_{P}(1)-X_{A}(1)||+\cdot+\left|D_{\min }-\right| X_{P}(N)-X_{A}(N)||$

so that the optimum is realized at a certain distance between the pair.

- The objective function The objective function is represented by

$$
F\left(X_{P}, X_{A}\right)=D\left(X_{P}, X_{A}\right)+\left(\Theta\left(X_{P}\right)+\left(1-\Theta\left(X_{A}\right)\right) .\right.
$$

Thus the value of the objective function approaches to zero, when the pair come very closely, while the particle is located in the supposed semi-algebraic or semi-analytic set and the antiparticle is not. One may dare to nominate this optimum as pair-annihilation the insinuation of which will be commented later.

- Personal best. The personal best value of a pair (with respect to the objective function) is in each time step renewed by the comparison of the current value and the past personal best.

- Group bests. The Group bests are represented by the record of the pioneering pairs: if the value of the objective function at a pair (the optima of which is zero) sinks below the threshold, the pair is recorded as a member of the group best set. It may happen that some members of the group best set are overcrowded around particular places; it would be troublesome, demanding the extra storage of the data, and causing the vague depiction of the boundary around the less populated places. In order to shun this, one might cull out the pairs which are located too close to the others in the registration of the group best set, or one might selectively do the registration so that the newcomer does not take its place immediate to the precursors. This treatment will determine the "resolution" of the depicted boundary. Once the pair is registered in the group best set, it should be subject to the pair-annihilation: that is to say, the pair is exempted from the time evolution of the group, and its position should be fixed thereupon.

- Solidification of frontier As for the solidification of the frontier by accumulating the group bests, the algorithm adopt a trick, as is used in the computation of trade-off curves[];in each time-step, one of the pairs in the frontier is chosen randomly and the attraction from it is taken into the updating of the velocities, so that The wandering pairs are drifted to the frontiers. (In fact, the chosen pair acts as the single "group best" in the usual PSO.) This sort of wanderersfrontiers interaction would admit several variants: the attractive or 
the repulsive: the long-ranged or the short ranged. One can switch them gingerly.

Under these preparations, the equation of the time evolution is represented by the equation and the work-tableau.

1. Initialization-of-Pairs

2. - Initialization-of-personal-best

3. - Initialization-of-group-best

4. while $\mathrm{i}<$ Number of iterations

5. - Update-velocity

6. - Update-position

7. - Evaluation-of-the-objective-function

8. - Update-personal-best

9. - Update-group-best (Solidification-of-frontier)

10. $-\mathrm{i}:=\mathrm{i}+1$

11. end while

\section{$3 \quad$ Numerical experiment}

In this section, I present the two kinds of simple computations. One of them is the result the two-dimensional case and the parameter setting in the computation is discussed. Another is the result of the four-dimensional case, in which the penalty function is slightly modified as an extension to the basic algorithm.

Let us see the two-dimensional case. The figures show the result of the numerical experiment applied to a simple semi-algebraic set. We assume the attraction between particle-and antiparticle pair, and we omit the interaction between the individual pairs and the center of the group. The profile of the boundary is fairly well delineated. In the first figure, the distribution of the pairs is shown. We see that a lot of pairs do not still come together around the boundary. However, in our formalism, the minimum of the objective function is zero. So we might remove the pairs which would not satisfy the criterion which is related to the objective function.

Fig. 1 shows the computation for the semi-algebraic set defined by

$$
\begin{aligned}
& x^{2}+y^{2} \leq 0 \\
& y \leq \frac{20}{4} x^{3}-3 x .
\end{aligned}
$$

It holds the stagnated pairs which ceased to move, although they are not caught at the boundary. These pairs are inadmissible. In Fig. 3, the profile of the system after the removal of inadmissible pairs is shown: the boundary becomes clearer.

The effect of the interaction toward the wandering pairs from the pairs at the boundaries might be seen in these computations. In Fig.3, the interaction is omitted. The depicted boundary becomes less clear because 
it is severed in some places. In Figure 5, the pairs settle at the boundary expels the wanderers who come close. In Fig. 6, we executed the computation with a slightly different criterion: the particle-antiparticle pair, among which the boundary intervenes, should be stabilized at a certain fixed distance. This figure shows the result of the removal of inadmissible pairs: it depicts the inner and the outer part of the semi-algebraic set. 


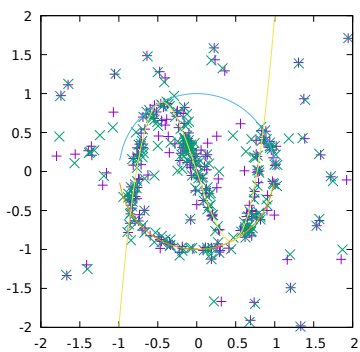

Figure 1: The algorithm explained in the article. This figure shows all of the pairs after the simulation of 800 steps.

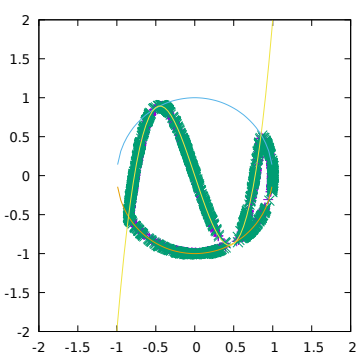

Figure 2: The depicted boundary. From the Fig. 1 the pairs (admissible as the solutions) are elicited. 


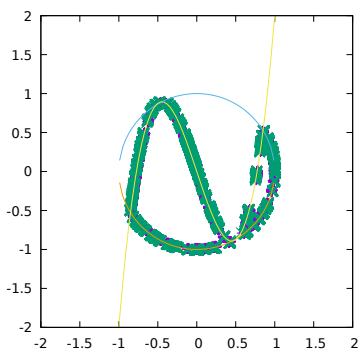

Figure 3: The interaction from the pairs at the boundary omitted. The other conditions are the same as those in Fig.3.

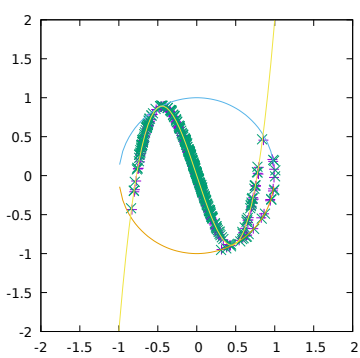

Figure 4: The interaction from the boundary is long-ranged, without cut-off. 


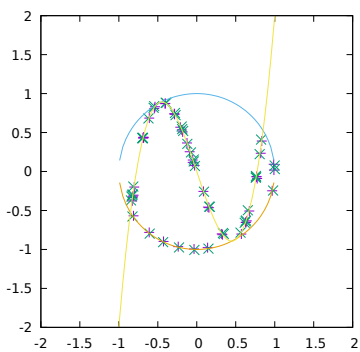

Figure 5: Every pair, already settled at the boundary, expels the wanderers who come closer. The other conditions are the same as those in Fig. 3.

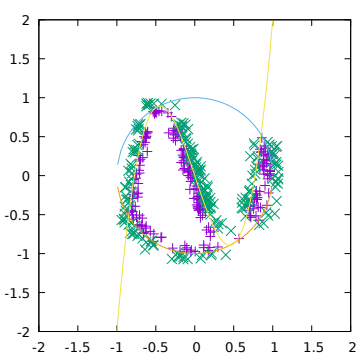

Figure 6: The pairs settle themselves at the boundary, keeping a certain distance between two members. The other conditions are the same as those in Fig. 3. 

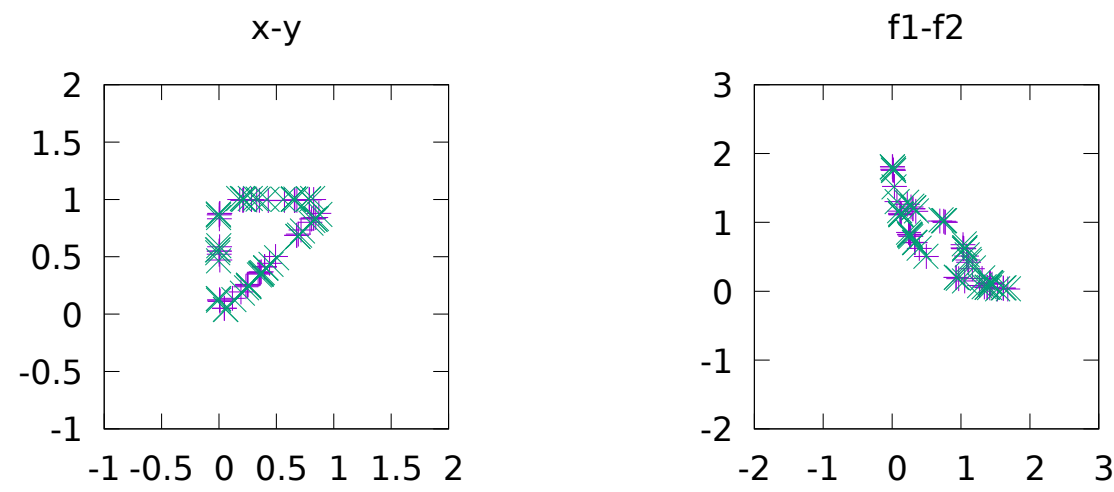

Figure 7: A computation in the four-dimensional space. The features in $\mathrm{x}-\mathrm{y}$ and f1-f2 planes are separately depicted.

Next, let us see the four-dimensional case. Fig. 7 shows the computation for the semi-algebraic set defined by

$$
\begin{aligned}
& f 1=x^{2}+y^{2} \leq 0 \\
& f 2=(x-1)^{2}+(y-1)^{2}, \\
& 0 \leq x \leq 1,0 \leq y \leq 1, x \leq y .
\end{aligned}
$$

The objective function is given by

$$
\begin{aligned}
& f\left(x_{P}, y_{P}, f 1_{P}, f 2_{P}, x_{A}, y_{A}, f 1_{A}, f 2_{A}\right) \\
= & \left\|f 1_{P}-x_{P}^{2}-y_{P}^{2}\right\| \\
& +\left\|f 2_{P}-\left(x_{P}-1\right)^{2}-\left(y_{P}-1\right)^{2}\right\| \\
& +\Theta\left(0 \leq x_{P} \leq 1,0 \leq y_{P} \leq 1, x_{P} \leq y_{P}\right) \\
& +\left\|f 1_{A}-x_{A}^{2}-y_{A}^{2}\right\| \\
& +\left\|f 2_{A}-\left(x_{A}-1\right)^{2}-\left(y_{A}-1\right)^{2}\right\| \\
& +\Theta\left(0 \leq x_{A} \leq 1,0 \leq y_{A} \leq 1, x_{A} \leq y_{A}\right) \\
& +D\left(X_{P}\left(x_{P}, y_{P}, f 1_{P}, f 2_{P}\right), X_{A}\left(x_{A}, y_{A}, f 1_{A}, f 2_{A}\right)\right) .
\end{aligned}
$$

The boundary by this semi-algebraic set is well depicted by the present algorithm. The first two terms in the penalty function are the extended 
part to the basic algorithm and they act as the restriction of equations. In this way, the present algorithm can coop with equations.

\section{Discussion}

The aim of the presented method is to depict the boundary of a semialgebraic set. Instead of normal PSO, it employs the pairs of particle and antiparticles. The particle should be placed in the points which belong to the semi-algebraic set, while the antiparticle should be placed out of the set. The objective function is designed in such a way that the minimum is zero, at which the particle and the anti-particle unite so that the unite pairs would depict the boundary. As the minimum of the objective function is zero, the algorithm could be robust, because it has only to find the expected zero. The sampling particle which would not come close to the boundary could be removed so that the boundary should be clearly depicted. The algorithm is as effective as normal PSO because the former adopts the effective components from the latter. However, the efficiency is mainly the result of the two-body interaction the particle-anti-particle; the guide from the group intelligence (such as the attraction/repulsion from the already settled pairs at the boundary) will be used for the regulative purpose, for example, in order to avoid the overpopulation at certain parts of the boundary.

I must point out that there are two types of boundaries. Now we consider only the real semi-algebraic set. It would cut the boundary in the real Euclidean space. On one side of the boundary, the real solutions always exist; but how will be the points in another side? They might not be described by the any real SEMI-ALGEBRAIC SET. For example, let us consider the problem.

The semi-algebraic set is given by

$\mathrm{f} 1=\mathrm{x} 1 * \mathrm{x} 1+\mathrm{x} 2 * \mathrm{x} 2$ 八 $\mathrm{f} 2=(\mathrm{x} 1-1) *(\mathrm{x} 1-1) 八 0<=\mathrm{x} 1<=1$ 八 $0<\mathrm{x} 2<=1$

How will be the boundary of this semi-algebraic set when it is projected to $(\mathrm{f} 1, \mathrm{f} 2)$ plain?

The solution is as follows:

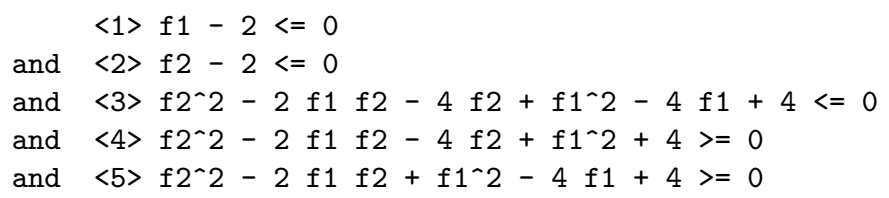

Those quadratic curves are originated from the edge and the section in the square in $(\mathrm{x} 1, \mathrm{x} 2)$ plane as follows

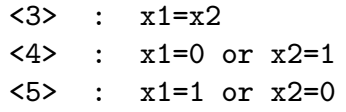

The corresponding algebraic set is specified by three algebraic curves. Let us retrieve the value of $(\mathrm{x} 1, \mathrm{x} 2)$ by solving the set of the equation with given (f1,f2). In the neighborhood of two curves (No.4 and No.5), 
in both sides of the boundary always exist the real solutions for $(\mathrm{x} 1, \mathrm{x} 2)$. (It is possible that $(\mathrm{x} 1, \mathrm{x} 2)$ does not locate in the square.) In contrast, in one side of the curve (No.3) (which is located above the segment $\mathrm{x} 1=\mathrm{x} 2$ ), the solutions $(\mathrm{x} 1, \mathrm{x} 2)$ are complex-valued and inappropriate as the point in the real four-dimensional space $(\mathrm{x} 1, \mathrm{x} 2, \mathrm{f} 1, \mathrm{f} 2)$. As for the curves No.4 and No.5, the particle-antiparticle pairs could seize the boundary if their $(\mathrm{x} 1, \mathrm{x} 2)$ coordinate components are located inside and outside the square respectively. However, for the curve No.2. such convenient formations fail to happen.

Would this mean that our scheme, by which the boundary is seized by the particle and antiparticle which moves outside and inside, is powerless in this case?

So long as we are able to process the polynomials by the symbolic computation, there is no problem in depicting the boundary, because we have only to process the polynomials in the (f1-f2) space. However, I propose the present algorithm as an expedient way to avoid the costly symbolic computations. Now it seems that we could not place the particle pairs outside and inside of the boundary, as the algorithm demands. However, this guess is groundless. We make use of the objective function which returns 0 or 1 according to the true or false status of the answer to the given problem. For example, it is possible that the particle is placed at the points $|f 1-x 1|<e$ and $|f 2-x 2|<e$; the anti-particle is placed at the complementary part in the Euclidean space.

In fact, in order to solidify the boundary of this sort, the algorithm for depicting trade-off curves might be more effective. Here I only insist that the present algorithm could also be applicable to this problem.

There is another expedient way. For example, let us shrink the range of $\mathrm{x}$ :

$\mathrm{f} 1=\mathrm{x} 1 * \mathrm{x} 1+\mathrm{x} 2 * \mathrm{x} 2$ 八 $\mathrm{f} 2=(\mathrm{x} 1-1) *(\mathrm{x} 1-1)$ 八 $0<=\mathrm{x} 1<=\mathrm{t} / 0<\mathrm{x} 2<=1$

The algorithm can draw the curve (of the periphery of a deformed fan) for each definite $t$. Likewise, by partitioning the range of $(x 1, x 2)$ into some sections we might solve the new problem by the present algorithm and we might guess the boundary of the initial problem.

In fact, the algorithm of drawing the trade-off curve would be effective for this problem. Such algorithms always push out the sample particles from inside and stop the particles if they touch at the boundary. However, there could be another scheme( which is a variant of our scheme), by pulling with each other between inside and outside. In fact, for this problem, there is an expedient way. Let us place the particle-antiparticle pairs:

Particle $\quad:(\mathrm{x} 1, \mathrm{x} 2, \mathrm{x} 1 * \mathrm{x} 1+\mathrm{x} 2 * \mathrm{x} 2,(\mathrm{x} 1-1) *(\mathrm{x} 1-1)+(\mathrm{x} 2-1) *(\mathrm{x} 2-1))$

Anti-particle: (x1,x2, A, , -A)

The coordinate point $(\mathrm{x} 1, \mathrm{x} 2)$ should be included in the square, which is given by $0<=x_{1}<=1$ and $0<x_{2}<=1$; the parameter $\mathrm{A}$ is assigned to each pair and keep fixed during the computation, and it should be uniformly distributed in a certain range. The object function for a pair is the distance: $\|x(P)-x(A)\|$. The particles $(\mathrm{P})$ finally come to locate at 


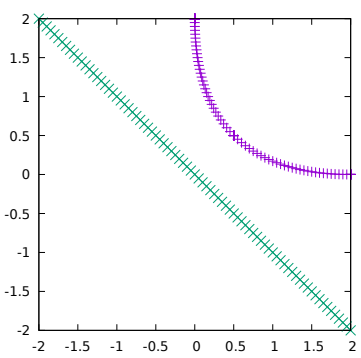

Figure 8: The drawing of the trade-off curve, by means of the present algorithm The upper curve and the lower line include the final locations of particles and antiparticles in the f1-f2 plane.

the curve $\left[f_{2}^{2}-2 f_{1} f_{2}-4 f_{2}+f_{1}^{2}-4 f_{1}+4<=0\right]$ in (f1,f2) plane, because each partner achieves the shortest distance. The result of the computation is shown in Fig. 8

Finally, we should comment on the possibility of collaboration from a genetic algorithm or other stochastic methods. In the examples presented above, the particle-antiparticle pairs are coupled at the initialization of the computation and the partners in a pair remain unchanged. However we can permit them to divorce and couple with others so that the computation would be more effective; for example, the stagnated couple (which is located in an inappropriate place) should be refreshed by being separated and by finding new partners. Such planning of re-marriage might be executed GA.

\section{Conclusion}

In this article, a variant of PSO algorithm is proposed and investigated as the method to depict the boundary of the semi-algebraic set. As the principal difference from normal PSO, it employs the pairs of particle and antiparticles. As the minimum of the objective function is zero, the algorithm could be robust and the correct solutions could be picked up easily from the sampling particle pairs. However, there are several topics, as are given in the former section, toward which the algorithm should be reinforced. We hope that future studies will achieve possible improvements.

\section{References}

[1] J.C.Kennedy, R.C.Eberhart, "Particle Swarm Optimization", in Proceedings of IEEE International Conference on Neural Networks, Perth, Australia, IEEE Service Center, Piscataway, NJ (1995), pp.1942-1948.

[2] Saptarsi Sengupta, Sanchita Basak, Richard Alan Peters II, "Particle Swarm Optimization: A survey of historical and recent developments 
with hybridization perspectives", in "Machine Learning and Knowledge Extraction", Multidisciplinary Digital Publishing Institute, Vol 1 (2018), pp157-191,

[3] Yu Lui, Zheng Qing in, Zhewen SHi, and Junying Chen, "Rule DIscovery with Particle Swarm Optimization", C.H-. Chi and K.-Y. Lam(Eds.) AWCC 2004, LNCS 3309, Springer (2014), pp. 291-296, .

[4] Feredico Marini, Beata Walczak, "Particle swarm optimization (PSO). A tutorial", Chemometrics and Intelligent Laboratory Systems 149 (2015) pp.153-165.

[5] Majid Jaberipour, Esmail Khorram, Behrooz Karimi, "Particle swarm algorithm for solving systems of nonlinear equations", Computers and Mathematics with Applications 62 (2011), pp. 566-576.

[6] Han Huang, Hu Qin, Zhigeng Hai, Andrew Lim, "Example-based learning particle swarm optimization for continuous optimization", Information Sciences 182(2012), pp.125-138.

[7] Mitchell Lane, Bing Xue, Ivy Liu, and Mengjie Zhang, "Gaussian Based Particle Swarm Optimisation and Statistical Clustering for Feature Selection", C.Blum and G.Ocha (Eds.): EvoCOP 2014, LNCS 8600, Springer (2014), pp. 133-144.

[8] Harish Garg, "A hybrid PSO-GA algorithm for constrained optimization problems", Applied Mathematics and Computation 274(2016), pp. 292-305.

[9] Atie Rachmad Syulistyo, Dwi M J Purnomo, Muhammad Febrian Rachmadi, and Adi Wibowo, "PARTICLE SWARM OPTIMIZATION (PSO) FOR TRAINING CONVOLUTIONAL NEURAL NETWORK(CNN)", Jurnal Ilmu Komputer dan Informasi (Journal of Computer Science and Information). 9/1(2016), pp. 52-58.

[10] Jonathan E. Fieldsend, "Optimizing decision trees using multiobjective particle swarm optimization", in "Swarm intelligence for multi-objective problems in data mining", Springer(2009), pp. 93-114.

[11] D. S. Arnon, G. E. Collins, S. McCallum, "Cylindrical Algebraic Decomposition I: The Basic Algorithm", Purdue University, Computer Science Technical Reports, (1982) 82-427.

[12] S. McCallum, "Solving Polynomial Strict Inequalities Using Cylindrical Algebraic Decomposition", The computer journal, Vol. 36, No.5 (1993), pp. 432-438.

[13] H. Hong, M. S. El-Din, "Variant Real Quantifier Elimination, Algorithm and Application", Journal of Symbolic Computation, vol 47,(7), (2012), pp.883-901.

[14] C. Chen, M. M. Maza, "Quantifier Elimination By Cylindrical Algebraic Decomposition Based on Regular Chains", ISSAC2014 ACM 978-1-4503-2501-1/14/07.

[15] HTTP://www.usna.edu/CS/oqepcad/B/QEPCAD.html 